\title{
Redes sociales en al sector educativo: Posicionamiento de un blog para el mercadeo de programas académicos
}

Jorge Iván Martínez Espitia ${ }^{1}$

\begin{abstract}
Resumen
Este artículo es producto de la investigación realizada en el marco del proyecto de investigación. Las redes sociales cuentan hoy con más de un billón de usuarios en todo el mundo y un blog es una alternativa que permite aumentar la visibilidad web de una empresa que mercadee bienes o servicios en las comunidades virtuales, convirtiéndolo en una alternativa gratuita y sencilla para los empresarios y a la vez confiable e interactiva para los usuarios que consultan y toman decisiones de compra de bienes o servicios por este medio ${ }^{2}$.

Este proyecto, corrobora que un blog puede ser útil para la promoción de los servicios de las instituciones educativas que, como la UNAD Florida, cuentan con un presupuesto limitado para mercadear sus programas académicos.
\end{abstract}

Palabras clave: mercadeo en redes sociales, comercio en educación, instituciones de educación a distancia, economía en la educación, opciones educativas, diseño de procedimientos de mercadeo. 


\title{
Social networks in the education sector: Positioning a blog for marketing academic programs
}

\begin{abstract}
This article is the result of research conducted within the framework of the research project to qualify for the degree of Master of Business Administration in UNAD Florida. Social networks now have over one billion users worldwide and a blog is an alternative that can increase the web visibility of a company that mercadee goods or services in virtual communities, making it a free and easy alternative for entrepreneurs and yet reliable and interactive for users who consult and make decisions to purchase goods or services through this medium.

This project confirms that a blog can be useful for promoting the services of educational institutions as, UNAD Florida, have a limited budget for marketing their academic programs.
\end{abstract}

Keywords: Social Media marketing. Educational Economics. Distance Teaching Institutions. Economics of Educations. Educational Options. Marketing Design procedures.

Recibido: 16-04-2014

Aceptado: 08-06-2014

\section{Introducción}

Han pasado 35 años desde la creación de las comunidades virtuales y es posible observar su efecto en la forma como hoy se concibe el marketing. Las redes sociales en internet representan un cambio en la manera de búsqueda de los bienes o servicios. Los clientes toman decisiones apoyándose en estas redes, con el fin de formarse una opinión, sobre la base del contenido y el entorno virtual en el cual se recibe la información.

Es posible aprovechar el marketing online en instituciones que produzcan bienes o presten servicios, en la medida que tiene muchas ventajas y beneficios: es gratuito, interactivo, masivo, con resultados rápidos y efectivos, colocando la empresa o institución en un lugar visible en la web. Este artículo se elaboró 
con el propósito de mostrar cómo mejorar la visibilidad web de una institución educativa que posee pagina institucional, a través de un blog.

Con este propósito se elaboró un blog que mostró un perfil de los servicios que ofrece la Universidad UNAD Florida, cuidando su diseño y aplicándole los procesos de optimización para los motores de búsqueda, a este blog luego se le realizó una evaluación formal que permitió establecer el efecto de estas comunidades virtuales, en el campo del marketing online.

El investigador indagó acerca de la visibilidad web de la UNAD Florida, ya que esta institución universitaria cuenta con su página institucional, es así como en Junio 25 de 2013 al utilizar Google como motor de búsqueda para encontrar programas de maestría en los Estados Unidos, el investigador no encontró, en esa oportunidad, en los resultados de las consultas a la UNAD Florida. A pesar de que esta institución está en internet, en esa oportunidad los programas que ofrece no estaban visibles en la red (Google, motor de búsqueda, 2013).

Teniendo en cuenta lo anterior se planteó la siguiente pregunta de investigación: ¿Cómo puede un blog ayudar a mejorar la visibilidad web de la UNAD Florida? De la cual surgió la pregunta que se respondió con esta investigación: ¿Cómo se puede posicionar un blog para ayudar a mejorar la visibilidad web de la UNAD Florida?

Esta investigación analizó la utilidad que puede dar el emplear un blog que contenga enlaces a la página web institucional empresarial. El análisis del diseño, el contenido y las estrategias de posicionamiento de $e$-marketing, pueden brindar información útil sobre el blog como una herramienta que incrementa la visibilidad de una institución o empresa en la web.

Esta investigación se justifica porque en la actualidad no existe literatura acerca de la evaluación formal de un blog elaborado con el objetivo de mejorar la visibilidad web de una empresa, sea del sector comercial o del área de los servicios.

La UNAD Florida, no aparece en los resultados de los motores de búsqueda con las palabras clave que pueden utilizarse en una consulta de los programas de maestría que oferta, entonces el blog http://mbaunadflorida.blogspot.com/, surgió con la idea de aportarle a la universidad más visibilidad en la web.

En el transcurso del trabajo se plantearon las siguientes preguntas de investigación:

¿Cómo ha sido evaluado el contenido y diseño del blog http://mbaunadflorida. blogspot.com que trata de mejorar la visibilidad web de una institución universitaria como es el caso de la UNAD Florida? 
¿Cuál fue el comportamiento del posicionamiento del blog http://mbaunadflorida. blogspot.com cuando se le aplicó el proceso SEO (Search Engine Optimization)?

¿Cuáles son los datos encontrados por la herramienta de Google Analytics para las personas que entran a investigar en el blog http://mbaunadflorida.blogspot. com?

\section{Historia del mercadeo en comunidades virtuales}

Los informáticos aficionados War Christensen y Randy Suess (1978), inventan el sistema Bulletin Board System (BBS) para informar a sus amigos sobre reuniones, hacer anuncios y compartir información. El blogging comenzó en 1995, el mismo año de la creación de Google y ya en 1998 se lanza la plataforma Blogger. Dentro de esta breve reseña histórica, se debe tener en cuenta que en el año 2004, nace Facebook, en el 2005 se lanza YouTube, en 2006 nace Twitter y en 2011 se lanza Google+, entre las opciones más reconocidas, siendo así que en el año 2012 las ventas por concepto de comercio asociado a las redes ascienden al orden de los 4.7 billones de dólares anuales y se estima que para el año 2017 esta cifra se incrementará hasta los 11 billones de dólares anuales.

\section{Social Media Marketing}

El marketing en medios sociales o Social Media Marketing (SMM) combina los objetivos de marketing en internet con medios sociales como blogs y muchos otros. El objetivo es por medio de estas herramientas mantener los productos y servicios en la mente de los consumidores de forma positiva, en definitiva, que los usuarios hablen bien de estos productos (Cano, 2013). La llamada web 2.0 moviliza a millones de medios de comunicación de comunidades virtuales (blogs, redes sociales y foros). A través de estos nuevos medios o micromedios surge una nueva forma de comunicación masiva que se ha empezado a denominar como auto-comunicación, entendida como la capacidad personal de los individuos y ciudadanos de difundir sus propios mensajes sin necesidad de mediación o intermediación de los medios tradicionales de comunicación de masas. Según Nutney (2010), se estima que hacia el año 2015 habrá unos 1.971,2 millones de usuarios de las redes sociales en todo el mundo y esta es una población potencial atractiva en la cual un proyecto debe realizar las acciones que le correspondan para mejorar su visibilidad ante estos futuros clientes.

Expertos en el tema del social media marketing como Casas (2013), que explica que las redes sociales como los blog, aumentan el tráfico, la visibilidad web y las ventas en internet y García E. (2013), que explica cómo los blog junto a las páginas web son sinónimos de mayor presencia en la red, son los que permiten establecer que un blog aumenta la visibilidad web. El investigador propone que 
si la página institucional de la UNAD Florida no aparece en los resultados de búsqueda de programas de maestría en los Estados Unidos, el blog creado para este efecto, se evalúe para determinar qué características debe tener un sitio que pueda aumentar la visibilidad web de una institución universitaria como es el caso de la UNAD Florida.

\section{Mercadeo en redes sociales}

Estupinian (2013), demostró que existe una relación directa entre el número de personas que visitan un website y la oportunidad de venta de un producto o servicio, esto puede asimilarse con la cantidad de estudiantes que luego de informarse, se inscriban a los programas que ofrece la universidad. Para una universidad sería muy grave no mantener inscripciones, puesto que se puede poner en peligro su supervivencia (Monti, 2006) y este aspecto podría ser resuelto en parte, con los beneficios que pueda dar un blog.

El futuro global en la educación virtual muestra un panorama que se puede aprovechar, un mercado creciente. Merril Lynch, la compañía norteamericana de análisis económico, plantea que a nivel global 160 millones de personas tomarán programas virtuales para el año 2025 (Barron, 2004).

Anivel mundial muchas empresas han aprovechado la posibilidad del e-marketing con el fin de asegurarse las primeras posiciones en los buscadores con lo que pueden obtener más ventas o nuevos clientes, que buscan productos específicos, y el uso de los blogs permite reunir varios intereses para obtener beneficios, ya que en estos se privilegia las novedades con un diálogo directo entre la empresa y el cliente; un blog asociado con enlaces directos al sitio web de la empresa contribuye en gran medida a posicionar mejor el sitio web empresarial.

Es en la internet que la comunidad virtual busca información acerca de los programas de maestría (Eced, 2011), en Europa y en general en todo el mundo, los futuros estudiantes, consultan en internet antes de tomar la decisión de elegir la escuela a la que ingresarán a seguir su programa de estudio (Del Amo, 2013), y al encontrar información que les sea útil en un portal universitario, es posible que alguno siga el programa de su preferencia en esta universidad (Yaq, 2013).

Las personas toman decisiones de adquirir un bien o servicio con la información que buscan en internet, pero además de esto es muy importante saber que les da más confianza la información que encuentran en la plataforma de las comunidades virtuales, como los blogs (Bourgeois, 2013). 


\section{El blog}

Es un sitio web en el que uno o varios autores publican cronológicamente textos o artículos, apareciendo primero el más reciente, el editor del blog conserva siempre la libertad de dejar publicado lo que crea pertinente y suele ser habitual que los propios lectores participen activamente a través de sus comentarios.

De acuerdo con García E. (2013), un blog debe ser una herramienta que permita una relación fluida entre quien lo escribe y quien lo lee. Entre sus características más relevantes están que sea de contenido original, se utilicen un contenido audiovisual adecuado, debe ser cuidadoso con la ortografía y respetuoso de las fuentes. Un blog que mejore la visibilidad web de una institución o empresa debe usar vínculos a las páginas web a las cuales se desea direccionar al lector por medio de pequeñas presentaciones, algunos utilizan animaciones, links, que al navegar le dirigen al sitio que se desea.

Un blog tiene características que hacen de él un proyecto atractivo, sobre todo porque se observan resultados en un tiempo relativamente corto y son gratuitos. Las instituciones que tienen un presupuesto limitado pueden utilizar esta herramienta, entre otras, que les permita desarrollar otra alternativa para el mercadeo de sus productos.

El blog toca temas complementarios que interesen de manera alternativa a posibles estudiantes de los programas online que oferta la universidad, es decir, a un futuro estudiante, puede parecerle interesante las opiniones de quienes ya han tomado el programa e interesarse en informarse del programa que ofrece la UNAD Florida y acceder también a su página institucional a través de los enlaces colocados en el blog, lo que promociona la página institucional de la universidad (Álvarez, 2011).

Ahora bien, si se crea un blog con este propósito, es importante tratar de conseguir que esté en la primera página de Google, para esto se debe optimizar la presentación interna del blog y algunos aspectos básicos a tener en cuenta son: publicar artículos útiles, cada página debe tener título y descripción únicos, generar contenido de calidad, integrar la página con redes sociales, introducir imágenes y enlaces, como lo señala Cárdenas (2013).

Por lo anterior, creado el blog, es necesario evaluar esta herramienta, para ello se utilizará una rúbrica, que analice el aspecto del diseño y contenidos del blog y a la vez se puede utilizar la herramienta Google Analytics para determinar la utilidad del proceso SEO (Search Engine Optimization) aplicado al blog mbaunadflorida.blogspot.com. 


\section{Proceso SEO (Search Engine Optimization)}

El proceso SEO (Search Engine Optimization), estudia estrategias para mejorar la visibilidad de un sitio web con el fin de ocupar las primeras páginas de los resultados de los motores de búsqueda. Una página web, de manera tradicional suele contener siempre la misma información. El contenido en sí mismo no se actualiza regularmente, lo que lo hace pasivo a los motores de búsqueda. En esta era de la información, estar bien posicionado se logra con base en un contenido dinámico y de calidad, esto es lo que atrae a posibles clientes, de esta forma es posible ser la elección número uno de quien solicita el servicio que se ofrece (Ventura, 2011).

Si una página web es pasiva, la alternativa es un blog, los motores de búsqueda son afines por páginas que interactúen con otras redes y en las cuales se muestre un gran contenido de información sobre un tema concreto, por lo que hacer un blog no solo posiciona, sino que posiciona muy bien, además es una forma gratuita de hacer SEO.

Los expertos opinan que para tener una mejor visibilidad online, las empresas deben rodearse de una comunidad comprometida en las redes sociales para mostrar la marca y para interactuar con los demás clientes o seguidores ganándose la confianza de los usuarios con estrategias participativas y en estas comunidades virtuales encontramos el blog, una herramienta de la web donde se decide, expone y controla los temas a tratar y la comunicación es de uno para muchos (Rivas, 2013).

\section{¿Cómo se hace más visible un blog en la web?}

Es fundamental, tener un plan para poder conformar con éxito un blog, tener una estrategia y a partir de ahí comenzar a desarrollar el proyecto, ¿Cuál puede ser esta estrategia para que el blog sea una herramienta eficaz para ayudar en las tareas comerciales? Pensar en el blog como si fuera la oficina, el contenido debe ser de calidad, pero estos artículos deben estar bien elaborados para que sean un imán para los clientes y tener todo a mano es importante. Esta investigación utilizó Blogger de Google ya que esta empresa ofrece la posibilidad de manejar otros productos útiles para este propósito desde una sola cuenta, entre ellos Google Grupos, Google Libros, Gmail, Google Maps, Google Earth, Hangouts, Google Drive, Picasa, Google Calendar, YouTube, Google Chrome, Google Plus, Google Play, Google AdSense, Google Adwords, Google Chromebook y están en desarrollo Google Summer of Code y Googleplex.

Luego de crear una cuenta en Blogger, se inicia el blog, el encabezado debe ser atractivo para que invite a leer un poco más y hacia la parte lateral no debe recargarse con tantos distractores que confundan al lector (Martínez, 2013). 
El fondo debe ser claro y las letras oscuras, se debe colocar algún artículo tipo incentivo y una foto, para darle identidad, los contenidos deben ser originales, mínimo de 300 palabras, utilizando palabras clave y sinónimos de estas y es importante ser frecuente en la producción de contenidos (Jiménez, 2013).

Para realizar la búsqueda de las palabras claves, la herramienta en Blogger es Google AdWords, una sencilla interfaz, donde de acuerdo a datos básicos como la descripción de lo que se piensa comercializar, datos básicos de la empresa, país e idioma, provee información en tiempo real de las palabras clave con alto, mediano o bajo tráfico, lo que permite un estudio de la competencia y la toma de medidas para que la página consultada, aumente la visibilidad web (Support. Google.com, 2013).

Lo básico para aparecer en los motores de búsqueda es otorgar el permiso requerido, por lo general esta configuración viene activada pero se recomienda darle un vistazo. Conociendo o no de programación lo recomendable es dejarse guiar por las herramientas que ofrece el sistema, se debe colocar foto y enlaces desde nuestro perfil en Google + hacia el blog, con la cuenta de herramientas para Webmasters de Google se puede averiguar el tráfico al sitio, consultando las palabras claves que han dado lugar a la aparición del blog en las búsquedas y algo muy importante: activar un Mapa de Sitio, en el que Google indexa las búsquedas y las hace más ágiles para ganar presencia en los resultados arrojados por el motor de búsqueda (González, 2013).

Utilizar las redes sociales es muy importante, esta investigación usó la red Google +. Este servicio fue lanzado en 2011 y cuenta en la actualidad con más de 300 millones de usuarios activos, mercado potencial en el que se incursiona cada vez que se haga una publicación y se comparta por este medio, lo que ayuda al SEO y aumenta el tráfico.

La herramienta Google Adsense, puede usar la publicidad online como una forma de atraer nuevos usuarios al sitio web, pero es verdad que Google es muy exigente en cuanto a cumplir con los preceptos de que el sitio tenga un contenido relevante y original, así como la transparencia y la facilidad de navegación por el mismo (Google Adsense, 2013).

\section{Evaluación del blog}

Gatica-Lara y Uribarren-Berrueta (2012), quienes son docentes de la Universidad Nacional Autónoma de México, afirman que en un blog se debe evaluar una gama completa de criterios, más allá de lo cuantitativo y cualitativo, lo más adecuado es utilizar una rúbrica y de manera específica que si se valora la calidad, la comprensión o el dominio de un tema se debe utilizar la rúbrica global. El esquema puede ser propuesto por el estudiante y este debe ser un 
instrumento de trabajo, siempre con el fin de elevar la calidad del proceso, esto de acuerdo al Documento Rúbrica para elaborar rúbricas del Buck Institute for Education (Mullinix, 2013).

Swag (2012), Cortez (2013) y Mata (2013), propone plantillas para la evaluación del contenido educativo, los parámetros corresponden en general a la identidad, la claridad de la intención del blog, el diseño, el contenido, el estilo, el tiempo, los enlaces, los debates y un análisis de la audiencia. Además es importante fijarse en las etiquetas, las entradas con fotos, enlaces o videos.

La evaluación del diseño y contenidos del blog mbaunadflorida.blogspot.com iniciaron con la validación de la rúbrica suministrada en el portal de Educación Mediática y Aplicación Didáctica de las TIC's (Marín, 2011) y diligenciada posteriormente por una muestra no probabilística de expertos.

La evaluación del posicionamiento y la demografía de los visitantes al blog mbaunadflorida.blogspot.com se realizó con datos obtenidos al usar la herramienta Google Analytics para medir el proceso SEO (Search Engine Optimization), se recopiló información con periodicidad semanal; se observaron y analizaron datos sobre el tráfico del sitio web, cual contenido es el más visitado, cuál es el promedio de visitas de la página y la hora local de las visitas, visualizar de que país llegan las visitas al sitio, que lenguaje hablan, de donde viene el tráfico. La información proporcionada por Google Analytics es muy importante, porque al conocerla, se pueden tomar decisiones de marketing más acertadas, para convertir los visitantes en clientes (Google Analytics, 2013).

\section{Materiales y métodos}

La investigación es de tipo exploratorio y cuantitativa. En la actualidad la literatura disponible muestra que no hay una investigación formal acerca de los aspectos de diseño, aplicación del proceso de optimización de motores de búsqueda y demografía de los visitantes al blog elaborado, que puedan establecer si un blog puede aumentar la visibilidad web de una empresa o institución (Hernández, 1997).

Esta investigación probó que un blog es una alternativa para mejorar la visibilidad web de una empresa o institución, utilizando como ejemplo el blog mbaunadflorida.blogspot.com, un blog que pretende ser útil para aumentar la visibilidad web de la UNAD Florida. Para ello se apoyó en las siguientes variables que fueron objeto de medición:

*Variable dependiente:

De acuerdo con Huerta (2012), el posicionamiento del blog tiene esta característica y es de interés principal en esta investigación. El análisis 
Redes sociales en al sector educativo: Posicionamiento de un blog para el mercadeo de programas académicos. Artículo producto de la investigación.

de esta variable da respuesta a la pregunta de investigación ¿Cuál fue el comportamiento del posicionamiento del blog mbaunadflorida.blogspot.com cuando se le aplicó el proceso SEO (Search Engine Optimization)? Para efectos de esta investigación la variable se denominó Posicionamiento Web del Blog mbaunadflorida.blogspot.com. Por los valores establecidos su valor está dado en números enteros, característica propia de una variable discreta, porque son los resultados arrojados por el motor de búsqueda Google de acuerdo a las palabras clave que se utilicen (Calderón, 2012).

\begin{tabular}{|c|c|c|}
\hline Palabras clave & Competencia & $\begin{array}{c}\text { Promedio de búsquedas } \\
\text { mensuales }\end{array}$ \\
\hline $\begin{array}{c}\text { Maestría online bilingüe } \\
\text { Colombia }\end{array}$ & Alta & 5.460 \\
\hline Maestría Bilingüe USA & Alta & 4.590 \\
\hline $\begin{array}{c}\text { Maestrías online florida } \\
\text { Master's online USA }\end{array}$ & Alta & 4.950 \\
\hline $\begin{array}{c}\text { Maestrías virtuales Estados } \\
\text { Unidos }\end{array}$ & Alta & 5.280 \\
\hline Promedio XX' & Alta & 5.170 \\
\hline
\end{tabular}

Tabla 1. Palabras clave encontradas para el blog mbaunadflorida.blogspot.com Fuente: Google Adwords. Tomado el 02 de julio de 2013.

*Variable independiente:

En esta investigación se determinó denominar como independiente la variable Palabras clave, porque tiene la propiedad de incidir en las demás variables objeto de estudio de esta investigación y fue alterada a lo largo de este estudio.

\section{*Variables intervinientes:}

Para dar respuesta a la pregunta de investigación ¿Cómo ha sido evaluado el contenido y diseño del blog mbaunadflorida.blogspot.com que trata de mejorar visibilidad web de una institución universitaria como es el caso de la UNAD Florida? Se establecieron las variables intervinientes: Objetivos del blog mbaunadflorida.blogspot.com, Gramática y ortografía del blog mbaunadflorida. blogspot.com, Frecuencia de publicación, Cantidad de hiperenlaces del blog mbaunadflorida.blogspot.com, Calidad de hiperenlaces del blog mbaunadflorida.blogspot.com, Títulos del blog mbaunadflorida.blogspot.com, 
Contenidos del blog mbaunadflorida.blogspot.com, Interés de los artículos del blog mbaunadflorida.blogspot.com, Extensión de los artículos del blog mbaunadflorida.blogspot.com, Comentarios del blog mbaunadflorida.blogspot. com e Inclusión de materiales visuales del blog mbaunadflorida.blogspot.com.

Para dar respuesta a la pregunta de investigación ¿Cuáles son los datos encontrados por la herramienta de Google Analytics para las personas que entran a investigar en el blog mbaunadflorida.blogspot.com?, se establecieron las siguientes variables intervinientes: Total de visitas al blog mbaunadflorida.blogspot.com, que es una variable continua, Entrada más visitada del blogmbaunadflorida. blogspot.com, como variable discreta; Duración media de la visita al blog mbaunadflorida.blogspot.com, como variable discreta; Porcentaje de retorno al blog mbaunadflorida.blogspot.com, que es una variable discreta; Fuente de tráfico al blog mbaunadflorida.blogspot.com, como variable discreta, Idioma de origen de los visitantes al blog mbaunadflorida.blogspot.com, como variable discreta y País de origen de los visitantes al blog mbaunadflorida.blogspot.com como variable discreta.

\section{Instrumentos}

La evaluación del diseño del blog se hizo por medio de una rúbrica. Las mediciones del posicionamiento del blog se realizaron utilizando medidas periódicas en las 15 semanas de toma de datos que fueron ordenados y tabulados. La herramienta Google Analytics, permitió medir de manera exacta las variables descritas que fueron objeto de análisis del blog mbaunadflorida.blogspot.com en las 15 semanas de la investigación.

Para la evaluación del diseño del blog se usó una rúbrica de acuerdo al modelo por propuesto por Núñez (2013), se solicitó a 5 docentes de la UNAD Florida, 5 estudiantes de los programas de maestría de la UNAD Florida y un experto en multimedia y educación evaluar el blog de acuerdo a la rúbrica mencionada, de forma que los evaluadores fueran por lo menos 3. Los datos encontrados se tabularon y graficaron (ver Tabla 2, Evaluación del diseño del blog mbaunadflorida.blogspot.com).

Para la medición del posicionamiento, se incorporó al blog el proceso SEO de manera gradual, y su fin fue mejorar la visibilidad web del blog. Para todo el proceso SEO, se usó la cuenta de Google. En el sitio Herramientas para Webmasters de Google, se activó el sitemap con el fin de indexar las páginas del blog y hacerlo accesible a los robots de búsqueda (Guisasola, 2012). Al ingresar a este sitio de herramientas también se realizó una configuración de preferencias activando la casilla para aceptar aparecer en los motores de búsqueda (García, 2013), se insertó una imagen a la entrada del blog para que aparezca en los resultados de búsqueda utilizando la herramienta Google Structured Data, se creó un perfil en Google + añadiendo este icono al blog (González, 2012), se 
optimizó el título del blog utilizando headings o encabezamientos, permalink y una descripción de búsqueda (Andrés, 2013). Luego de la aplicación de este proceso se tomaron 15 muestras de los resultados que se obtienen en el motor de búsqueda Google al usar las palabras clave que la herramienta Google Analytics muestra que son las que las personas usan para encontrar programas de maestría.

El método de análisis de datos para dar respuesta a las preguntas de investigación utilizó la estadística descriptiva. Se hizo la correlación de la variable dependiente Posicionamiento web del blog mbaunadflorida.blogspot.com con la variable independiente Palabras clave. Para tabular la información el investigador asignó una puntuación de 5 a la variable interviniente Palabras clave, que muestre el resultado del blog mbaunadflorida.blogspot.com en la primera página del motor de búsqueda Google; 4 si lo muestra en la 2 página; 3 si se muestra en la 3 página; 2 si lo muestra en la 4 pagina, 1 si aparece en la página 5 y 0 si aparece de la página 6 en adelante.

Para ordenar los datos, es decir, la distribución de frecuencias, se elaboraron tablas de frecuencias e histogramas. También se encontró para estas preguntas de investigación, como la más importante medida de tendencia central, la moda, es decir el resultado de la búsqueda de más frecuencia durante el estudio.

El método de análisis de datos para dar respuesta a la preguntas de investigación ¿Cómo ha sido evaluado el contenido y diseño del blog mbaunadflorida.blogspot. com que trata de mejorar visibilidad web de una institución universitaria como es el caso de la UNAD Florida? Determinó que la evaluación del diseño del blog, también tendrá una puntuación entre 1 y 5 en los criterios evaluados. Para analizar los datos hallados en las variables que responden esta pregunta de investigación se utilizó la Escala de Likert, propuesta por Hernández (1997), con el fin de correlacionar la actitud de los evaluadores frente a lo observado por ellos en el blog y valorado con las puntuaciones: (5) Totalmente adecuado, (4) Adecuado, (3) Ni adecuado ni no adecuado, (2) Indeciso pero más bien adecuado, (1) Indeciso pero más bien no adecuado y (0) Totalmente inadecuado.

Para ordenar los datos de las pregunta de investigación, es decir, la distribución de frecuencias, se graficaron en una tabla de frecuencias e histogramas. Se encontraron las medidas de tendencia central. La que se más utilizó para este análisis de datos fue la media aritmética o promedio.

\section{Resultados}

Se establecieron dos tipos diferentes de fuentes de datos de acuerdo con las preguntas de investigación: una primera fuente de de los datos que dio respuesta a la pregunta de investigación ¿Cómo ha sido evaluado el contenido y diseño del blog mbaunadflorida.blogspot.com que trata de mejorar visibilidad 
web de una institución universitaria como es el caso de la UNAD Florida? Consistió en establecer una muestra no probabilística, que es un tipo apto para investigaciones exploratorias. De manera cuidadosa y controlada se eligieron personas con conocimientos y experiencia en educación online es decir una muestra de expertos, sea como docentes o estudiantes de programas de maestría y haciendo el tamaño de la muestra (n) igual a 3. Se solicitó a estos, evaluar el blog mbaunadflorida.blogspot.com de acuerdo a la rúbrica mencionada y los datos que se encontraron se tabularon y graficaron.

Una segunda fuente de los datos que dio respuesta a las preguntas de investigación ¿Cuál fue el comportamiento del posicionamiento del blog mbaunadflorida. blogspot.com cuando se le aplicó el proceso SEO (Search Engine Optimization)? y ¿Cuáles son los datos encontrados por la herramienta de Google Analytics para las personas que entran a investigar en el blog mbaunadflorida.blogspot.com? Son las herramientas Google Adwords y Google Analytics, que son servicios gratuitos de estadísticas de sitios web, lo que ofrecen información agrupada según los intereses de las personas involucradas en el funcionamiento de una página de internet.

Se establecieron dos métodos para recolección de datos, que dieron respuesta a las preguntas de investigación: el método que recolectó los datos que dieron respuesta a la pregunta de investigación ¿Cómo ha sido evaluado el contenido y diseño del blog mbaunadflorida.blogspot.com que trata de mejorar visibilidad web de una institución universitaria como es el caso de la UNAD Florida? Consistió de manera inicial en validar la rúbrica, se contactó un experto en informática y multimedia en educación, la Doctora María del Rosario Sáenz Rodríguez, quién validó la rúbrica al considerarla confiable y apta. Se solicitó a estos, evaluar el blog mbaunadflorida.blogspot.com de acuerdo con la rúbrica mencionada y los datos que se encontraron se tabularon y graficaron.

La Validez Externa para esta pregunta de investigación, es decir la confianza en los resultados se logró porque no hay interacción previa entre los evaluadores y el blog, los evaluadores realizan esta labor sin influencias o direccionamientos.

La rúbrica validada por la Dra. Sáenz se envió con una solicitud de su diligenciamiento a 5 docentes de los programas de maestría de la UNAD Florida, 5 estudiantes del programa de maestría de UNAD Florida. También se solicitó a la Dra. Sáenz diligenciar esta evaluación, dada su experiencia en el área. Se dio como plazo para la recepción del documento diligenciado el 31 de octubre de 2013.

El otro método de recolección de datos que dio respuesta a las preguntas de investigación ¿Cuál fue el comportamiento del posicionamiento del blog mbaunadflorida.blogspot.com cuando se le aplicó el proceso SEO (Search Engine Optimization)? y ¿Cuáles son los datos encontrados por la 
herramienta de Google Analytics para las personas que entran a investigar en el blog mbaunadflorida.blogspot.com? Se establecieron 15 grupos de series cronológicas, con mediciones similares y equivalentes. Para lograr el control y la validez interna de estas preguntas de investigación, fueron emparejadas por medio de la variable Palabras clave que es la que ejerce más influencia sobre los resultados de la investigación al comparar los datos medidos. La validez externa para esta pregunta de investigación está dada por cuanto las mediciones cronológicas pueden ser susceptibles de repetición en cualquier momento.

El 2 de julio de 2013, se utilizó el planificador de Google Adwords, buscando las palabras claves que se consideraran para una universidad bilingüe, con modalidad online, ubicada en la Florida - Estados Unidos y Colombia, que ofrece programas de maestría. Se escogieron los primeros resultados que se listan a continuación y que conforman la variable independiente Palabras clave: Maestría online bilingüe Colombia, Maestría Bilingüe USA, Maestrías online Florida, Master's online USA, Maestrías Virtuales Estados Unidos. Comparten como característica que todas tienen una alta competencia y su promedio de búsqueda es de 5.090 veces por mes.

Los datos encontrados que dan respuesta a la pregunta de investigación ¿Cuál fue el comportamiento del posicionamiento del blog mbaunadflorida.blogspot. com cuando se le aplicó el proceso SEO (Search Engine Optimization)? son los que corresponden a la relación de la variable dependiente Posicionamiento web del blog mbaunadflorida.blogspot.com y la variable independiente Palabras clave. Corresponden a la serie cronológica de 15 mediciones semanales de las entradas al blog. Los valores hallados fueron: El valor de la variable interviniente Objetivos del blog mbaunadflorida.blogspot.com hallado fue 4,83. El valor de la variable interviniente Gramática y ortografía del blog mbaunadflorida.blogspot.com fue de 5. El valor de la variable interviniente Frecuencia de publicación fue de 5. El valor de la variable interviniente Cantidad de hiperenlaces del blog mbaunadflorida.blogspot.com fue de 5. El valor de la variable interviniente Calidad de hiperenlaces del blog mbaunadflorida. blogspot.com fue de 4,67. El valor de la variable interviniente Títulos del blog mbaunadflorida.blogspot.com fue de 4,33. El valor de la variable interviniente Contenidos del blog mbaunadflorida.blogspot.com fue de 4,67. El valor de la variable interviniente Interés de los artículos del blog mbaunadflorida.blogspot. com fue de 4,67. El valor de la variable interviniente Extensión de los artículos del blog mbaunadflorida.blogspot.com fue de 4,17. El valor de la variable interviniente Comentarios del blog mbaunadflorida.blogspot.com fue de 4,33. El valor de la variable interviniente Derechos de autor fue de 5. El valor de la variable interviniente Inclusión de materiales visuales del blog mbaunadflorida. blogspot.com fue de 4.33 . 


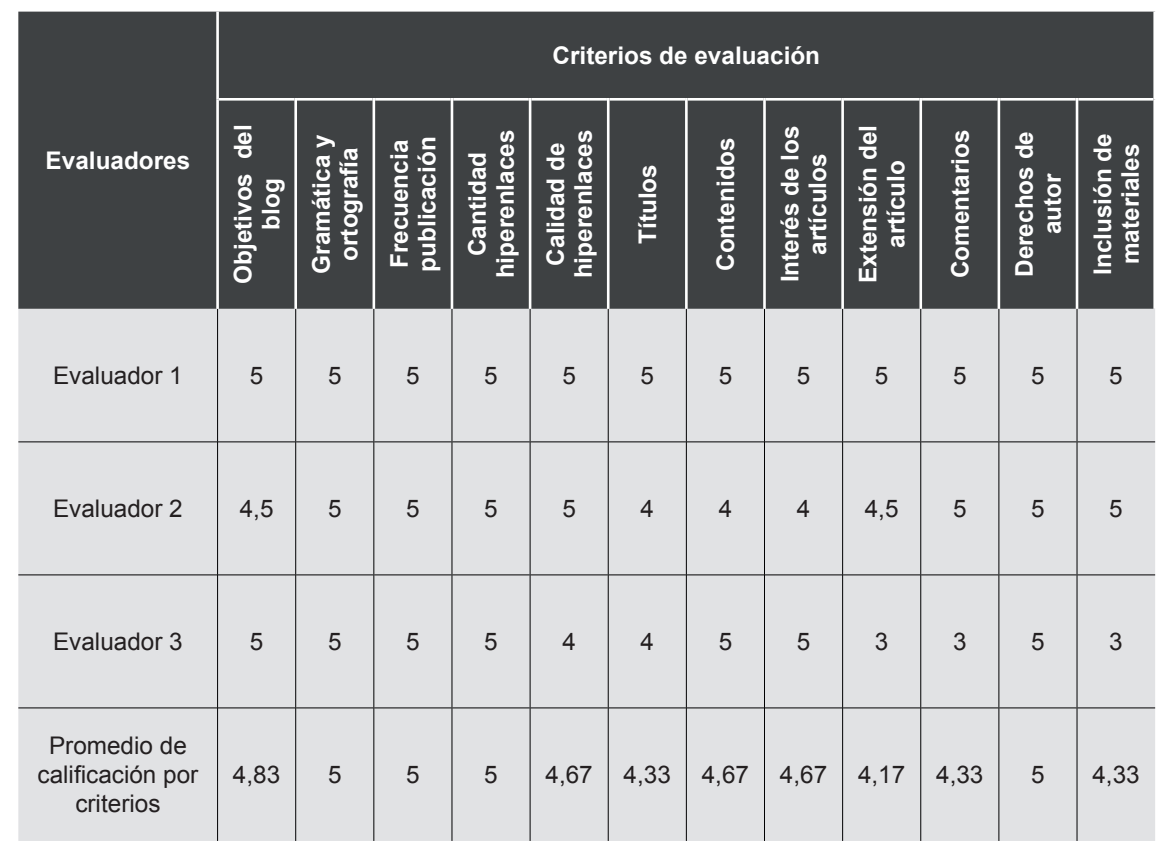

Tabla 2. Evaluación del diseño del blog mbaunadflorida.blogspot.com Fuente: Rúbrica diligenciada en octubre de 2013 por expertos ${ }^{2}$.

Los anteriores datos son producto de las mediciones realizadas de una parte por la muestra de expertos que evaluaron el diseño del blog y de otra parte por el investigador que realizó las series cronológicas de resultados del motor de búsqueda Google de acuerdo con las palabras clave y a los resultados arrojados por la herramienta Google Analytics, tomando en cuenta de manera estricta las tres preguntas de investigación.

Los datos hallados que dan respuesta a la pregunta de investigación ¿Cuáles son los datos encontrados por la herramienta de Google Analytics para las personas que entran a investigar en el blog mbaunadflorida.blogspot.com?, fueron: el valor de la variable interviniente Total de Visitas al blog mbaunadflorida. blogspot.com en este periodo es de 171; el valor de la variable interviniente Entrada más visitada al blog mbaunadflorida.blogspot.com es de $27 \%$ para la entrada ¿Cuánto cuesta una maestría online en Unad Florida?

2. Evaluador 1: María del Rosario Sáenz. Ingeniera de Sistemas, Especialista en Gerencia Informática, Especialista en Informática y multimedia en educación. Directora del departamento de sistemas del Colegio Montemorel. Asesora municipal de tecnologías en educación.

Evaluador 2: Harold Rivera, Ingeniero, con maestría en Gerencia de Ingeniería, Doctorado en Gerencia de Liderazgo organizacional. Es uno de los dos socios mayoritarios de Bussines Advisers Inc de Latinoamérica.

Evaluador 3: Mariluz Beltrán. Contadora Pública. Especialista en revisoría fiscal. Aspirante a la Maestría en Administración de Negocios de la UNAD Florida. 
El valor hallado para la variable interviniente Retorno al blog mbaunadflorida. blogspot.com fue de 63,8\% para los nuevos visitantes y de 36,2 para los que retornan al blog. El valor hallado para la variable interviniente Fuente de Tráfico al blog mbaunadflorida.blogspot.com, es decir el modo en el que los visitantes ingresan al blog muestra que el mayor porcentaje $56 \%$ proviene de redes sociales. El valor encontrado para la variable interviniente Duración media de la visita al blog al blog mbaunadflorida.blogspot.com es de alrededor de 1 minuto. Para la variable interviniente Idioma de origen de los visitantes al blog mbaunadflorida. blogspot.com se encontró que un $89 \%$ es el español, incluidos los hispanos de los Estados Unidos. Para la variable interviniente País de origen de los visitantes al blog al blog mbaunadflorida.blogspot.com, un 75\% provienen de Colombia, sin embargo, un porcentaje importante de visitantes el 9\%, proviene de los Estados Unidos.

\section{Discusión}

Los datos se analizaron utilizando el método de estadística descriptiva para variables. La moda, es decir el valor asignado con mayor frecuencia para los atributos de la variable fue de 5, por lo que de acuerdo con la escala de Likert significa que el diseño es en su gran mayoría totalmente adecuado. La media aritmética hallada para esta variable fue de 4,67, con base en la escala de Likert significa que en promedio el contenido y diseño del blog mbaunadflorida. blogspot.com se encuentra en un rango entre adecuado y totalmente adecuado, para un blog que trata de mejorar la visibilidad web de una institución universitaria como es el caso de la UNAD Florida.

Para los datos encontrados que dan respuesta a la pregunta de investigación ¿Qué incidencia tiene el proceso SEO (Search Engine Optimization) en el posicionamiento del blog mbaunadflorida.blogspot.com?, se utilizo el método de estadística descriptiva para las variables. Para la variable dependiente Posicionamiento web del blog mbaunadflorida.blogspot.com se estableció que la moda, es decir el dato de posicionamiento de mayor frecuencia, corresponde al 2, y significa la cuarta página de resultados, pero en las 15 semanas del estudio, todas las búsquedas utilizando las 5 palabras clave objeto del análisis dieron como resultado 5, es decir el blog mbaunadflorida.blogspot.com se posicionó en la primera página de resultados. 


\begin{tabular}{|c|c|c|c|c|c|c|c|c|c|c|c|c|c|c|c|}
\hline & 㔬 & $\begin{array}{l}\overline{3} \\
\stackrel{2}{\sim}\end{array}$ & $\begin{array}{l}\overline{5} \\
\overline{7} \\
\stackrel{0}{2}\end{array}$ & $\begin{array}{l}\bar{j} \\
\text { d. }\end{array}$ & $\begin{array}{l}\stackrel{\circ}{8} \\
\stackrel{8}{8}\end{array}$ & \begin{tabular}{l} 
영 \\
\} $\\
{\text { ᄋ }}$ & $\begin{array}{l}\stackrel{\circ}{8} \\
\stackrel{8}{0}\end{array}$ & & 용 & $\begin{array}{l}\text { 융 } \\
\text { ळ } \\
\text { 용 }\end{array}$ & $\begin{array}{l}\text { ᄋ } \\
\text { क् } \\
\text { ஸ }\end{array}$ & $\begin{array}{l}\text { 유 } \\
\text { 오 }\end{array}$ & $\begin{array}{l}\stackrel{\circ}{\circ} \\
\text { స̃ } \\
\text { సે }\end{array}$ & 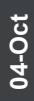 & tே요 \\
\hline $\begin{array}{c}\text { Maestría online } \\
\text { bilingüe Colombia }\end{array}$ & 0 & 0 & 0 & 0 & 2 & 2 & 2 & 2 & 2 & 3 & 3 & 4 & 4 & 5 & 5 \\
\hline $\begin{array}{c}\text { Maestría Bilingüe } \\
\text { USA }\end{array}$ & 0 & 0 & 0 & 0 & 2 & 2 & 2 & 1 & 3 & 2 & 2 & 2 & 2 & 4 & 5 \\
\hline $\begin{array}{c}\text { Maestrías online } \\
\text { Florida }\end{array}$ & 0 & 0 & 0 & 0 & 2 & 3 & 2 & 2 & 2 & 2 & 3 & 3 & 4 & 5 & 5 \\
\hline Master's online USA & 0 & 1 & 1 & 1 & 2 & 2 & 1 & 2 & 2 & 5 & 2 & 3 & 3 & 5 & 5 \\
\hline $\begin{array}{l}\text { Maestrías virtuales } \\
\text { Estados Unidos }\end{array}$ & 0 & 0 & 1 & 2 & 3 & 2 & 2 & 2 & 2 & 2 & 3 & 3 & 3 & 5 & 5 \\
\hline
\end{tabular}
\end{tabular}

Tabla 3. Secuencia cronológica de posicionamiento del blog mbaunadflorida.blogspot.com

Fuente: https://www.google.com.co/ 15 Series cronológicas de búsqueda, realizadas entre el 05 de julio de 2013 y el 11 de octubre de 2013.

Los valores encontrados en las mediciones se agruparon en histogramas de frecuencia determinando que el blog apareció en los resultados del motor de búsqueda Google por primera vez al utilizar las palabras clave en la segunda semana de medición y de ahí en adelante el progreso fue paulatino, en la décima semana de medición, el blog mbaunadflorida.blogspot.com llego a la primera página de resultados, al utilizar el motor de búsqueda Google, con la palabra clave Master's online USA.

Posterior a este hallazgo, en la semana 15 todas las búsquedas utilizando las palabras clave halladas para estudio del blog posicionaron el blog mbaunadflorida. blogspot.com en la primera página de resultados del motor de búsqueda Google, hallando que para el final del estudio, es decir en 15 semanas, se logró posicionar el blog mbaunadflorida.blogspot.com en la zona más visible de la web, dado que el $13,3 \%$ de los datos hallados corresponde al posicionamiento del blog mbaunadflorida.blogspot.com en la primera páginas al utilizar las palabras claves establecidas al inicio del estudio.

Con base en este análisis estadístico, es posible determinar que al aplicarle al blog mbaunadflorida.blogspot.com el proceso Search Engine Optimization (SEO), este incide de manera positiva en el posicionamiento web al utilizar el motor de búsqueda de Google, ubicándolo en la primera página de resultados al término de la semana 15 de mediciones.

Los datos encontrados que dan respuesta a la pregunta de investigación ¿Cuáles son las características de las personas que entran a investigar en el blog mbaunadflorida.blogspot.com?, se analizaron utilizando el método de estadística descriptiva para las variables.

El 36,2 \% de los visitantes que entraron al blog, retornaron a este, por lo que se estima que los elementos incluidos en el llamaron su atención de forma que les 
Redes sociales en al sector educativo: Posicionamiento de un blog para el mercadeo de programas académicos. Artículo producto de la investigación.

generó necesidad de una nueva visita. La búsqueda orgánica del blog, es decir las personas que de manera espontánea usaron las palabras clave y entraron al blog corresponden al $25 \%$, pero en este estudio tuvo gran peso la búsqueda originada en las redes sociales ya que un $56 \%$ tuvo acceso al blog por medio de la red social Google +, comportamiento compatible con los hallazgos del marco teórico, en el que las personas prefieren el Social Media Marketing, es decir que un sitio referido a través de las redes sociales genera mayor confianza en sus miembros.

Los visitantes en promedio duran 58 segundos en la visita al blog, usan el español en un 88,3\%, incluido un 14,62\% de hispanos ubicados en los Estados Unidos y también es importante resaltar del tráfico fue el 9,94\% de los visitantes que tienen el inglés como su lengua materna.

Aunque el 84,80\% provienen de Colombia y Estados Unidos, el blog es visitado desde otros países latinoamericanos en un 9,35\% y un 5,26\% de los visitantes provienen de Centroamérica, un visitante es decir el 0,58\% proviene de África. Estas personas consideraron importante la entrada ¿Cuánto cuesta una maestría online en UNAD Florida? Ya que un 25,7 \% ingresó en esta página y un 18,7\% se interesó en la Universidad, ya que visitó la entrada Conozcamos la UNAD Florida, Tablas 4 y 5 .

\begin{tabular}{|c|c|c|c|}
\hline Categoría & Frecuencias absolutas & Frecuencias relativas & Frecuencias acumuladas \\
\hline Idioma & Número de visitas & Número de visitas & Número de visitas \\
\hline Español & 126 & $73,68 \%$ & $73,68 \%$ \\
\hline Español en USA & 25 & $14,62 \%$ & $88,30 \%$ \\
\hline Inglés USA & 17 & $9,94 \%$ & $98,25 \%$ \\
\hline Francés & 3 & $1,75 \%$ & $100 \%$ \\
\hline Total & 171 & $100 \%$ & \\
\hline
\end{tabular}

Tabla 4. Distribución de frecuencias de la variable Visión general por idioma de los visitantes al blog mbaunadflorida.blogspot.com

Fuente: Google Analytics. Tomado el 15 de octubre de 2013. 


\begin{tabular}{|c|c|c|c|}
\hline $\begin{array}{c}\text { Categoría } \\
\text { País-Territorio }\end{array}$ & $\begin{array}{c}\text { Frecuencias absolutas } \\
\text { Número de visitas }\end{array}$ & $\begin{array}{c}\text { Frecuencias relativas } \\
\text { Número de visitas }\end{array}$ & $\begin{array}{c}\text { Frecuencias acumuladas } \\
\text { Número de visitas }\end{array}$ \\
\hline Colombia & 129 & $75,44 \%$ & $75,44 \%$ \\
\hline USA & 16 & $9,36 \%$ & $84,80 \%$ \\
\hline Ecuador & 7 & $4,09 \%$ & $88,89 \%$ \\
\hline Perú & 7 & $4,09 \%$ & $92,98 \%$ \\
\hline República Dominicana & 4 & $2,34 \%$ & $95,32 \%$ \\
\hline Bolivia & 2 & $1,17 \%$ & $96,49 \%$ \\
\hline Guatemala & 2 & $1,17 \%$ & $97,66 \%$ \\
\hline Panamá & 2 & $1,17 \%$ & $98,83 \%$ \\
\hline Curazao & 1 & $0,58 \%$ & $99,42 \%$ \\
\hline Marruecos & 1 & $0,58 \%$ & $100 \%$ \\
\hline Total & 171 & $100 \%$ & \\
\hline
\end{tabular}

Tabla 5. Distribución de frecuencias de la variable Visión general por país de los visitantes al blog mbaunadflorida.blogspot.com

Fuente: Google Analytics. Tomado el 15 de octubre de 2013.

\section{Conclusiones y recomendaciones}

Las comunidades virtuales son un lugar prometedor para el mercadeo de productos y servicios. Un blog puede por sí solo hacer el mercadeo de productos o servicios o ayudar en mejorar la visibilidad web de una empresa. Para alcanzar ese objetivo, se debe tener claro un plan de marketing que incluya el diseño del sitio, los procesos de mejoramiento de visibilidad web como el SEO y progresivamente el análisis de la información demográfica de los visitantes al sitio web que permita identificar en tiempo real las deficiencias del sitio y tomar los correctivos.

Las redes sociales son un elemento indispensable del web marketing; gracias a su direccionamiento, para el caso del blogmbaunadflorida.blogspot.com durante el tiempo de mediciones, 171 personas en un lapso de 15 semanas recibieron información de la UNAD Florida, desde sitios tan lejanos como el África o desde mercados tan prometedores como el hispano de los Estados Unidos. Esta investigación demostró que si se usan las técnicas adecuadas es posible posicionar un blog en el primer lugar y eso puede ser aplicable a cualquier producto o servicio.

Usar Google parece ser también parte del efecto positivo mostrado en esta investigación, la variedad de sus aplicaciones, lo hace una especie de plataforma integral de servicios, donde de manera gratuita se puede tomar lo necesario para conformar un proyecto, evaluarlo periódicamente y mejorarlo con el tiempo. 
Hay que ser enfático en eso, la posibilidad de interacción de estas páginas les imprime una dinámica importante que debe resolver con prontitud las inquietudes de los usuarios, creando canales cada vez más eficientes de comunicación que son los que marcan la diferencia con otras formas más rígidas de promoción de productos o servicios y estando atento a estos requerimientos es posible los procedimientos de mejora continua.

Este estudio experimental observó el comportamiento de los visitantes a un blog y crea un marco conceptual acerca del efecto de las redes sociales en el marketing actual en la visibilidad del producto; queda por definir el impacto en la decisión de compra de los usuarios, que motivados por la información de la página alternativa, consultan la pagina web institucional. ¿Bajo qué criterios estos toman la decisión de adquirir el bien o servicio? Esta pregunta abriría paso a otras líneas de investigación enmarcadas dentro de este interesante conglomerado que forman las comunidades virtuales.

\section{Referencias bibliográficas}

2013. Cómo elegir un máster en 6 pasos. Consultado el 19 de septiembre de 2013. Disponible en: http://www.expansion.com/2013/06/27/entorno/1372344367.html

2013. SEO para principiantes: Cómo mejorar la visibilidad en Google. Consultado el 30 de agosto de 2013.Disponible en: http://ecolisima.com/seo-para-principiantescomo-mejorar-visibilidad-googl/

Álvarez, M. 2011. ¿Por qué no aparece mi web en Google? Consultado el 12 de agosto de 2013. Disponible en: http://www.desarrolloweb.com/faq/mi-web-no-aparecegoogle.html

Andrés, D. 2013. 5 Consejos antes de publicar una entrada en blogger. Importante! Consultado el 13 de septiembre de 2013. Disponible en: http://www.terrenoblogger. co/2013/04/5-consejos-antes-de-publicar-una-entrada-blogger.html

Barron, H. 2004. Seis problemas de los sistemas universitarios de educación en Línea. Consultado el 19 de septiembre de 2013.Disponible en: http://www.um.es/ead/red/12/ barron.pdf

Bernal, C. 2006. Metodología de la investigación para administración, economía, humanidades y ciencias sociales. (pp 84-102). México: Pearson Educación.

Bicego, G. 2012. Palabras Clave: Donde y Como Usarlas Para Optimizar tu Posicionamiento Web. Consultado el 12 de agosto de 2013. Disponible en: http:// impulsandoideas.net/147/palabras-clave-posicionamiento-web/\#.UjCavNlyJWJ 
Bourgeois, M. 2013. Lo digital influenciando decisiones de compra. Consultado el 24 de septiembre de 2013. Disponible en: http://www.iab.com.uy/destacada/informe-denielsen-lo-digital-influenciando-decisiones-de-compra

Calderón, J. 2012. Clasificación de variables según la naturaleza de su medición $y$ fines operacionales. Consultado el 13 de agosto de 2013. Disponible en: http:// tesisdepostgradoperu.blogspot.com/2012/11/variables.html

Cano, C. 2013. ¿Que es un plan de social media marketing? Consultado el 20 de septiembre de 2013. Disponible en: http://comenzandodecero.com/que-es-un-plande-social-media-marketing/

Cárdenas, A. 2013. SEO para principiantes: Cómo mejorar la visibilidad en Google. Consultado el 21 de septiembre de 2013.Disponible en: http://ecolisima.com/seo-paraprincipiantes-como-mejorar-visibilidad-googl/

Casas, J. 2013. E-commerce: 7 estrategias para aumentar tráfico, visibilidad y ventas utilizando redes sociales. Consultado el 30 de agosto de 2013.Disponible en: http:// postcron.com/es/blog/e-commerce-7-estrategias-para-aumentar-trafico-visibilidad-yventas/

Cortez, G. 2013. Rubrica para evaluar entrada de blog. Consultado el 18 de septiembre de 2013. Disponible en: http://www.slideshare.net/GinisCortes/rubrica-para-evaluarentrada-de-blog

Dawson, C. 2013. ¿Que es posicionamiento orgánico? Consultado el 03 de septiembre de 2013. Disponible en: http://www.posicionamientowebenbuscadores.com/blog/ que-es-posicionamiento-organico/

Dean, R. 2013. ¿Cómo aumentar la visibilidad de mi Blog? Consultado el 22 de agosto de 2013.Disponible en: http://blogger3cero.com/como-aumentar-la-visibilidad-de-miblog/

Del Amo, E. 2013. FAQs o preguntas frecuentes para elegir carrera: Qué, cómo y dónde. Consultado el 03 de septiembre de 2013. Disponible en: http://www.aprendemas. com/Guias/EspecialElegirCarrera_Jun2012/P6.asp

Del Valle, E. 2013. Los 50 términos imprescindibles del Marketing Online, bien explicados (I). Consultado el 25 de agosto de 2013.Disponible en: http://www. socialmediaycontenidos.com/50-terminos-imprescindibles-marketing-online-bienexplicados-1

Eced, L. 2011. 7 Herramientas para evaluar tus Redes Sociales y tu blog. Consultado el 12 de septiembre de 2013. Disponible en: http://luiseced.wordpress.com/2011/12/28/7herramientas-evaluar-redes-sociales-blog/

Estupinian, W. 2013. ¿Pagerank? Como aparecer en los primeros lugares de Google? Consultado el 06 de agosto de 2013. Disponible en: http://www.xtrategico.com/blog. php?doc_id=11 
Redes sociales en al sector educativo: Posicionamiento de un blog para el mercadeo de programas académicos. Artículo producto de la investigación.

García, E. 2013. Social Media Blog, refuerza tu visibilidad. Consultado el 15 de septiembre de 2013. Disponible en: http://www.gestion.org/social-media/42965/ social-media-blog-refuerza-tu-visibilidad/

García, M. 2013. Ponle descripción a tus entradas en los resultados de los buscadores. Consultado el 04 de agosto de 2013.Disponible en: http://diariosdelanube.blogspot. com/2013/06/aprende-escribir-la-descripcion-de-tus.html\#.UICradlyJWI

Gatica, F. \& Uribarren, T. 2012. ¿Cómo elaborar una rúbrica? Consultado el 24 de septiembre de 2013. Disponible en: http://www.colegiomedizbolio.com.mx/site_2012/ wp-content/uploads/2013/08/Como-hacer-una-rubrica-10_PEM_GATICA.pdf

Gómez, M. 2006. Introducción a la metodología de la investigación cientifica. pp. 5962. Ed. Brujas. Córdoba.

González, J. 2012. Cómo mostrar tu foto junto a tu blog en los resultados de Google. Consultado el 21 de agosto de 2013. Disponible en: http://www.iniciablog.com/2012/11/ mostrar-foto-perfil-resultados-google.html.

González, J. 2013. ¿Qué es un blog? Consultado el 18 de septiembre de 2013.Disponible en: http://www.iniciablog.com/2013/04/que-es-un-blog.html

González, J 2013. Guía práctica sobre SEO en Blogger. Consultado el 04 de agosto de 2013.Disponible en: http://www.iniciablog.com/2013/04/guia-consejosposicionamiento-seo-blogger.html

Google Adsense. 2013. Uso de la publicidad online para atraer a nuevos usuarios al sitio. Consultado el 20 de septiembre de 2013. Disponible en: https://support.google. com/adsense/answer/1348727?hl=es\&ref_topic $=1348718$

Google Analytics. 2013. Introducción a Google Analytics. Consultado el 23 de agosto de 2013. Disponible en: https://support.google.com/analytics/answer/1008065?hl=es-419

Google, Motor de Búsqueda. 2013. Resultados para la búsqueda Maestría bilingüe Estados Unidos.

Google support. 2013. Optimización del motor de búsqueda (SEO). Consultado el 11 de agosto de 2013. Disponible en: https://support.google.com/webmasters/ answer/35291?hl=es-419

Guisasola, J. 2012. ¿Qué es un Sitemap y para qué sirve? Consultado el 27 de septiembre de 2013. Disponible en: http://quiwiq.com/posicionamiento/que-es-un-sitemap-ypara-que-sirve/941

Hernández, R. 1997. Metodología de la investigación. Consultado el 18 de septiembre de 2013. Disponible en: http://metodologiasdelainvestigacion.files.wordpress. com/2012/11/metodologia-investigacion-hernandez-sampieri-lucio.pdf 
Huerta, M. 2012. Operacionalización de variables. Consultado el 26 de septiembre de 2013. Disponible en: http://www.slideshare.net/moiseshuerta10/operacionalizacinde-variables-15233275

Ivirtual.info. 2008. ¿Qué es un Blog y para qué sirve? Consultado el 14 de agosto de 2013. Disponible en: http://www.ivirtual.info/articulos/2-sitios-web/7-ique-es-unblog-y-para-que-sirve.html

Jiménez, J. 2013. 10 factores básicos de SEO que debes conocer aunque no sepas SEO. Consultado el 10 de septiembre de 2013. Disponible en: http://3cero.com/factoresbasicos-seo/

Marín, V. 2011. Educación Mediática y Aplicación Didactica de las TIC's: Rubrica para la evaluación de blogs. Disponible en: htpp://edmetic.es

Márquez, A. 2012. 8 tips para aprender sobre posicionamiento web. Consultado el 30 de septiembre de 2013. Disponible en: http://www.posicionamientoenbuscadores-sl. com/8-tips-para-aprender-sobre-posicionamiento-web

Martínez, F. 2013. Cómo diseñar un blog que sea un imán para tus clientes. Consultado el 23 de septiembre de 2013.Disponible en: http://www.negociosenauge.net/comodisenar-un-blog-que-sea-un-iman-para-tus-clientes/

Mata, G. 2013. Rubrica para evaluar blog. Consultado el 19 de agosto de 2013. Disponible en: http://www.slideshare.net/licmata/rbrica-evaluar-blog

Monti. M. 2006. Puertas abiertas y creatividad para atraer estudiantes. Consultado el 22 de agosto de 2013. Disponible en http://www.lanacion.com.ar/812854-puertasabiertas-y-creatividad-para-atraer-estudiantes

Mullinix, B. 2013. Rúbrica para elaborar rúbricas. Consultado el 16 de septiembre de 2013. Disponible en: http://www.eduteka.org/rubrica2.php

Núñez, M. 2013. Rúbrica para la evaluación de blogs. Consultado el 12 de agosto de 2013. Disponible en: http://www.rafaelrobles.com/wiki/index.php?title=Blogs_ filos\%C3\%B3ficos

Nuttney, A. 2010. The Social Networking Market Opportunity. Consultado el 25 de septiembre de 2013. Disponible en: http://www.globalbusinessinsights.com/content/ rbtc0140m.pdf

Rivas, S. 2013. Diferencias entre Comunidad, Red Social, Foro y Blog. Consultado el 01 de septiembre de 2013. Disponible en: http://www.rivassanti.net/CommunityManager/Diferencias-entre-Comunidad-Red-Social-Foro-Blog.php

Support.google.com. 2013. ¿Qué es Google AdWords? Consultado el 15 de agosto de 2013. Disponible en : https://support.google.com/adwords/certification/ answer/1704410?hl=es\&ref_topic $=2799732$ 
Redes sociales en al sector educativo: Posicionamiento de un blog para el mercadeo de programas académicos. Artículo producto de la investigación.

Swag, T. 2012. Como evaluar un blog de los compañeros. Consultado el 29 de septiembre de 2013. Disponible en: http://swags-8.blogspot.com/2010/10/como-evaluar-un-blogde-los-companeros.html.

Toro, J. 2013. Web 2.0 ¿Que es y para qué sirve? Consultado el 24 de agosto de 2013. Disponible en: http://juanalonsotoro.com/web-2-o-que-es-y-para-que-sirve/.

Ventura, V. 2011. Como ganar visibilidad en internet. Consultado el 17 de septiembre de 2013. Disponible en: http://www.emprenderalia.com/como-ganar-visibilidad-eninternet/.

Villavicencio, I. 2012. Validez de un estudio. Consultado el 08 de agosto de 2013. Disponible en: http://www.slideshare.net/ilsejanine/validacion-de-instrumentos.

Wikipedia. 2013. Blog. Consultado el 23 de septiembre de 2013. Disponible en: http:// es.wikipedia.org/wiki/Blog.

Wikipedia. 2013. Posicionamiento en buscadores. Consultado el 15 de agosto de 2013. Disponible en: http://es.wikipedia.org/wiki/Posicionamiento_en_buscadores.

Yaq, P. 2013. Cómo elegir una universidad a tu medida. Consultado el 22 de septiembre de 2013. Disponible en: http://yaq.es/reportajes/como-elegir-universidad. 